\title{
OBJECT-ORIENTED PROGRAMMING FOR WIDE-AREA COMPUTING
}

\author{
Jayadev Misra \\ University of Texas at Austin \\ misra@cs.utexas.edu
}

Abstract Object-based sequential programming has had a major impact on software engineering. However, object-based concurrent programming remains elusive as an effective programming tool. The class of applications that will be implemented on future high-bandwidth networks of processors will be significantly more ambitious than the current applications (which are mostly involved with transmissions of digital data and images), and object-based concurrent programming has the potential to simplify designs of such applications. Many of the programming concepts developed for databases, object-oriented programming and designs of reactive systems can be unified into a compact model of concurrent programs that can serve as the foundation for designing these future applications.

There are several key questions in developing a model of concurrentobjects for wide-area computing. Foremost among the concerns is the difficulty associated with managing concurrent interactions, particularly when a multitude of machines interact. This is the main topic of this research. Additionally, issues of security and fault-tolerance are central to wide-area computing. How can a secure computation be carried out if the data and procedure reside at different machines? If a computation involves several thousand machines - as would be expected for a computation on the world-wide web - is it realistic to allow an unknown party to have exclusive access to a resource? That party may crash requiring a major roll-back effort. Is it reasonable to queue a caller for access to a resource when the resource manager could possibly fail, causing the caller to block? In this case, it may be more efficient to reject the call if the resource is not readily available. We seek a model that allows us to experiment with a variety of questions of this nature. 